\title{
The LHCb pixel hybrid photon detectors
}

\section{Robert W. Lambert* ${ }^{\dagger}$}

The University of Edinburgh

E-mail: R.W.Lamberteed.ac.uk

The Ring Imaging Cherenkov counters of the LHCb experiment use an innovative type of photon detector, the pixel Hybrid Photon Detector. The Cherenkov photons are detected by 484 such devices. The production of the photon detectors is now complete, and the devices have all been fully qualified in dedicated laboratory test facilities. The quantum efficiencies far exceed expectations, with peak values in excess of $30 \%$. We also present the measured ion feedback rates, dead/noisy pixel fractions and dark count rates.

International Workshop on new Photon-Detectors

June 27-29 2007

Kobe, Japan

\footnotetext{
* Speaker.

$\dagger$ On behalf of the LHCb RICH collaboration
} 


\section{Introduction}

The LHC beauty experiment (LHCb) [1, 2] will investigate CP-violation through careful examination of the nature of the decays of hadrons containing $b$-quarks at the Large Hadron Collider (LHC) at CERN. The Ring Imaging Cherenkov (RICH) system is crucial for the particle identification of charged kaons and pions within the LHCb experiment [3]. Two RICH detectors, employing three radiator materials, are used to cover the full momentum range. The upstream RICH detector, RICH 1, records the velocity of particles with low momentum, 2-60 GeV/c, the second RICH detector, RICH 2, records the velocity of particles with higher momentum, up to $100 \mathrm{GeV} / c$. The number of photons produced by each charged track with $\beta \simeq 1$ is for example $\sim 15$ from the $\mathrm{C}_{4} \mathrm{~F}_{10}$ radiator in RICH 1. Therefore we require single-photon sensitive devices across four photon-detector planes covering a total area of $\simeq 2.6 \mathrm{~m}^{2}$. To perform accurate particle identification within the LHCb RICH over the lifetime of the experiment, the light detectors must exhibit the requirements listed in Table 1 .

This paper describes the production and testing results for the entire sample of pixel Hybrid Photon Detectors (HPDs), the chosen single-photon detectors for the LHCb RICHes. We also briefly discuss the installation progress of the RICH system.

\section{Hybrid photon detectors}

HPDs are a technology exploiting the advantages of vacuum photon detector tubes with pixelated silicon detectors and read out with integrated circuit technology [4]. A quartz window transmits light from a broad wavelength range, extending into the UV, which falls upon a multi-alkali photocathode. The photocathode is biased to $-20 \mathrm{kV}$ relative to the silicon chip. Through the photoelectric effect in the photocathode, incident light is converted to photoelectrons which travel within the HPD vacuum and are accelerated toward the pixelated silicon detector. Fig. 1 shows example schematics. The photocathode is imaged and demagnified onto the encapsulated silicon sensor. The silicon sensor is bump-bonded [5] to a pixelised readout chip which is also encapsulated within the vacuum of the HPD, resulting in low capacitive load to the front-end electronics and hence a

Table 1: Selected requirements and parameters for the LHCb RICH photon detectors.

\begin{tabular}{ll|rl}
\hline Feature & Comment & Value & Unit \\
\hline Active area & -- & 80 & $\%$ \\
Detection range & wavelength & $200-600$ & $\mathrm{~nm}$ \\
Lifetime & at least & 10 & years \\
Hit rate & maximum & 50 & $\mathrm{MHz} \mathrm{cm}^{-2}$ \\
Dark count rate & maximum & 5 & $\mathrm{MHz} \mathrm{cm}^{-2}$ \\
Afterpulse probability & maximum & 1 & $\%$ \\
Granularity & -- & $2.5 \times 2.5$ & $\mathrm{~mm}^{2}$ \\
Clock frequency & LHC clock & 40 & $\mathrm{MHz}$ \\
Internal buffering & trigger latency & 4 & $\mu \mathrm{s}$ \\
Radiation tolerance & -- & 30 & $\mathrm{krad}$ \\
\hline B-field conditions & maximum & 5 & $\mathrm{mT}$ \\
& direction & random & \\
\hline
\end{tabular}




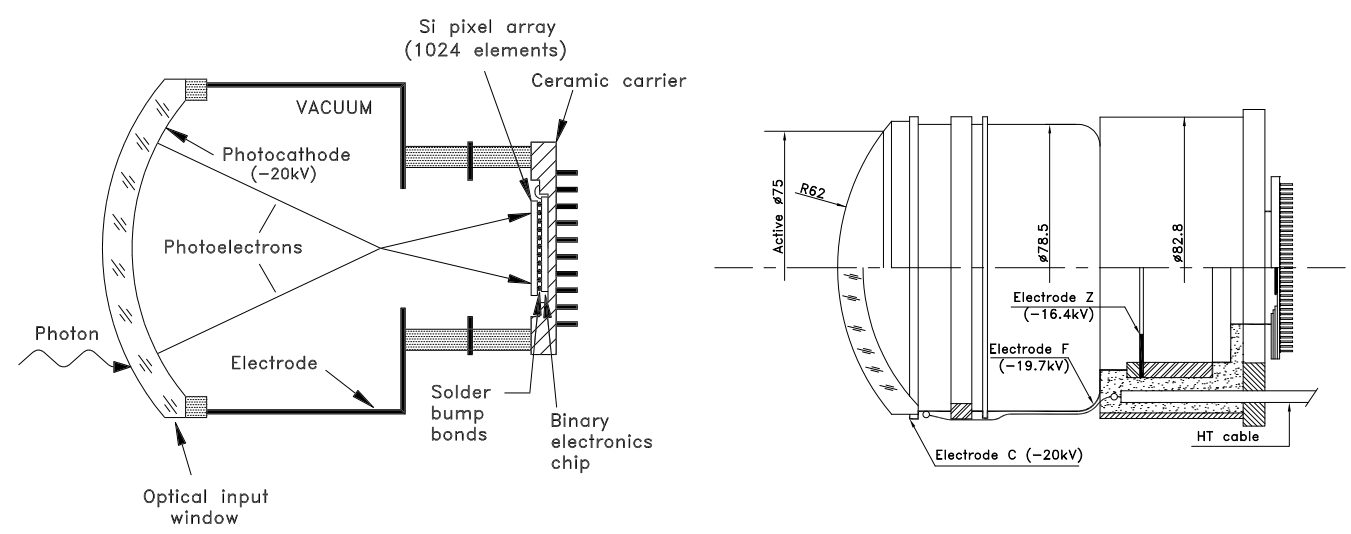

Figure 1: Hybrid Photon Detectors for the LHCb RICH [3]. Left, HPD schematic with electron optics highlighted. Right, more technical description of a complete HPD.

Table 2: Selected specifications for final assembled HPDs.

\begin{tabular}{ll|rrr|l}
\hline Feature & Comment & Lower & Typical & Upper & Unit \\
\hline Quantum efficiency & $270 \mathrm{~nm}$ & 20.0 & 23.3 & - & $\%$ \\
$(\mathrm{QE})\left(\eta_{q}\right)$ & $400 \mathrm{~nm}$ & 15.7 & 19.3 & - & $\%$ \\
& $520 \mathrm{~nm}$ & 8.3 & 10.7 & - & $\%$ \\
\hline Dark count & -- & - & 5.0 & - & $\mathrm{kHzcm}^{-2}$ \\
Afterpulsing & Ion feedback rate & - & - & 1 & $\%$ \\
Lifetime & $\leq 10 \%$ deviation & 10 & - & - & yrs \\
Radiation hardness & - & 30 & - & - & $\mathrm{krad}$ \\
Pixel threshold & -- & - & - & 1500 & electrons \\
Sensor leakage current & At $80 \mathrm{~V}$ at $50^{\circ} \mathrm{C}$ & - & 1.0 & - & $\mu \mathrm{A}$ \\
Photoelectron efficiency & - & - & 85 & - & $\%$ \\
Working channels & -- & 95 & - & - & $\%$ \\
\hline
\end{tabular}

high signal-to-noise ratio. The HPD pixel chip was a joint development between the LHCb and ALICE collaborations [6]. The detection and read-out chain within each HPD is pixelated to 8192 pixels, $0.5 \mathrm{~mm}$ by $0.0625 \mathrm{~mm}$ in dimension. Eight pixels are actively ORed together for operation in the LHCb experiment to form one 'super-pixel' of dimension $0.5 \mathrm{~mm}$ by $0.5 \mathrm{~mm}$, of which there are 1024. Each of the 8192 pixels consist of sensor, as well as individually configurable threshold, amplifier, data buffer and readout electronics.

A selection of the specifications for final assembled HPDs is given in Table 2. These are based on the parameters from Table 1 and the performance of prototype HPDs. LHCb has independently verified that manufactured HPDs are within specifications.

The HPDs are manufactured in several stages in an international effort in close collaboration with industry. LHCb was involved at all stages in the design and testing of the components. The details of the procedure are reported in [4]. The final stage of encapsulation of the HPD, photocathode deposition, vacuum sealing and potting was performed by Photonis-DEP ${ }^{1}$. We require 484 HPDs to equip both RICH detectors. The manufacture of production HPDs began in mid 2005 and

\footnotetext{
${ }^{1}$ Photonis Netherlands B.V., Dwazziewegen 2, P.O. Box 60, NL-9300 AB Roden, The Netherlands. Formerly Delft Electronic Products (DEP).
} 
we have now received the entire sample of 550 production HPDs, which also includes spares. We report on the test results for 548 HPDs, and 545 for quantum efficiency (QE) testing.

\section{Photo-detector testing}

\subsection{Methodologies and facilities}

Photonis-DEP test the HPDs following manufacture, before delivery, to characterise each HPD with respect to the specifications listed in Table 2 . This includes a full QE measurement performed on every manufactured HPD.

Two Photo-Detector Test Facilities (PDTFs), each with two test stations, were commissioned by LHCb to fully quantify the HPDs delivered by Photonis-DEP. At the PDTFs all functionality and specifications unique to each HPD are tested. This includes a confirmation measurement of the QE performed at seven wavelengths on a subsample of $10 \%$ of HPDs.

The afterpulse rate from ion-feedback is determined from a timing scan. As photoelectrons ejected from the photocathode travel through the vacuum of the tube they may ionise residual gas molecules. The positive ions produced are accelerated towards the photocathode. When the ions impact they deposit their energy and result in multiple electron emission. The electrons are then accelerated back to the anode, forming a large cluster of hits on the anode. Due to the crossfocusing of the HPD the highest electron density is close to the longitudinal centre, and in the lateral centre, of the HPD (demonstrated in Fig. 1). Ion feedback therefore has a characteristic delay resulting from the drift time of the ions. The ratio of delayed large clusters to the initial prompt signal is the ion-feedback probability and measures the vacuum quality of the HPD. Mechanisms for the introduction of residual gas involve diffusion through the quartz window, diffusion through imperfections in the tube body or the vacuum seal, and outgassing of the HPD internally.

\subsection{Results from the production sample}

We have tested and qualified all production HPDs at the PDTFs, and have measured only 12

Table 3: Selected results for production HPDs

\begin{tabular}{ll|rrl}
\hline Measurement & Comment & Mean & R.M.S. width & Unit \\
\hline Photocathode image & Radius & 6615 & 122 & $\mu \mathrm{m}$ \\
& offset & 386 & 207 & $\mu \mathrm{m}$ \\
\hline Dark count rate & $5 \mathrm{M}$ triggers & 2.54 & 5.98 & $\mathrm{kHz} \mathrm{cm}^{-2}$ \\
Afterpulse probability & ion feedback & 0.03 & 0.14 & $\%$ \\
Threshold & average each HPD & 1064 & 101 & $\mathrm{e}-$ \\
Noise & average each HPD & 145 & 12 & $\mathrm{e}-$ \\
Dead pixels & out of 8192 & 12 & 32 & pixels \\
Noisy pixels & out of 8192 & 2 & 14 & $\mathrm{e}-$ \\
Leakage current & Si sensor & 1492 & 2660 & $\mathrm{nA}$ \\
\hline Quantum & $270 \mathrm{~nm}$ & 30.9 & 3.3 & $\%$ \\
efficiency $\dagger$ & $400 \mathrm{~nm}$ & 24.3 & 2.3 & $\%$ \\
$(\mathrm{QE})\left(\eta_{q}\right)$ & $520 \mathrm{~nm}$ & 13.4 & 1.4 & $\%$ \\
\hline Integrated QE $\dagger$ & $\Sigma \mathrm{QE} \delta \mathrm{E}$ & 0.968 & 0.114 & $\mathrm{eV}$ \\
\hline
\end{tabular}

PDTF measurements, except for $\dagger$ which are Photonis-DEP measurements. Errors are not included. 

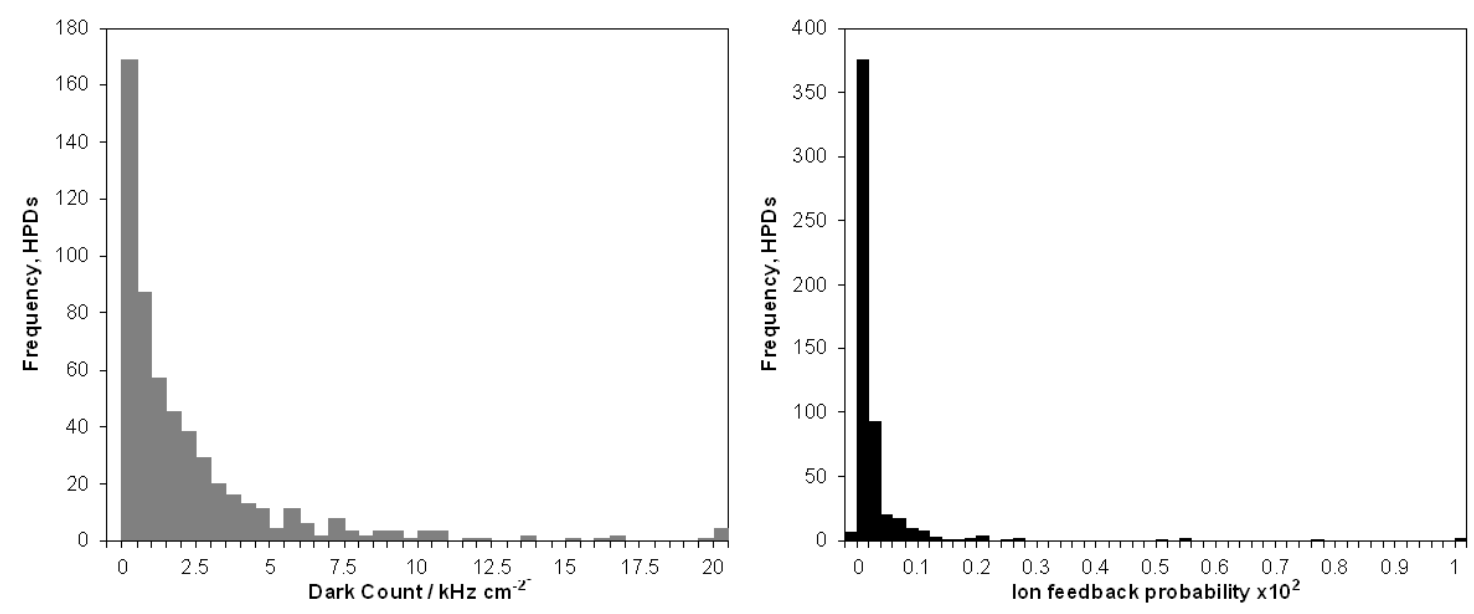

Figure 2: Noise and afterpulse results from production HPDs. Left, Dark count from 5M trigger samples. Right, ion-feedback probability $\left(\times 10^{2}\right)$ from timing scans.
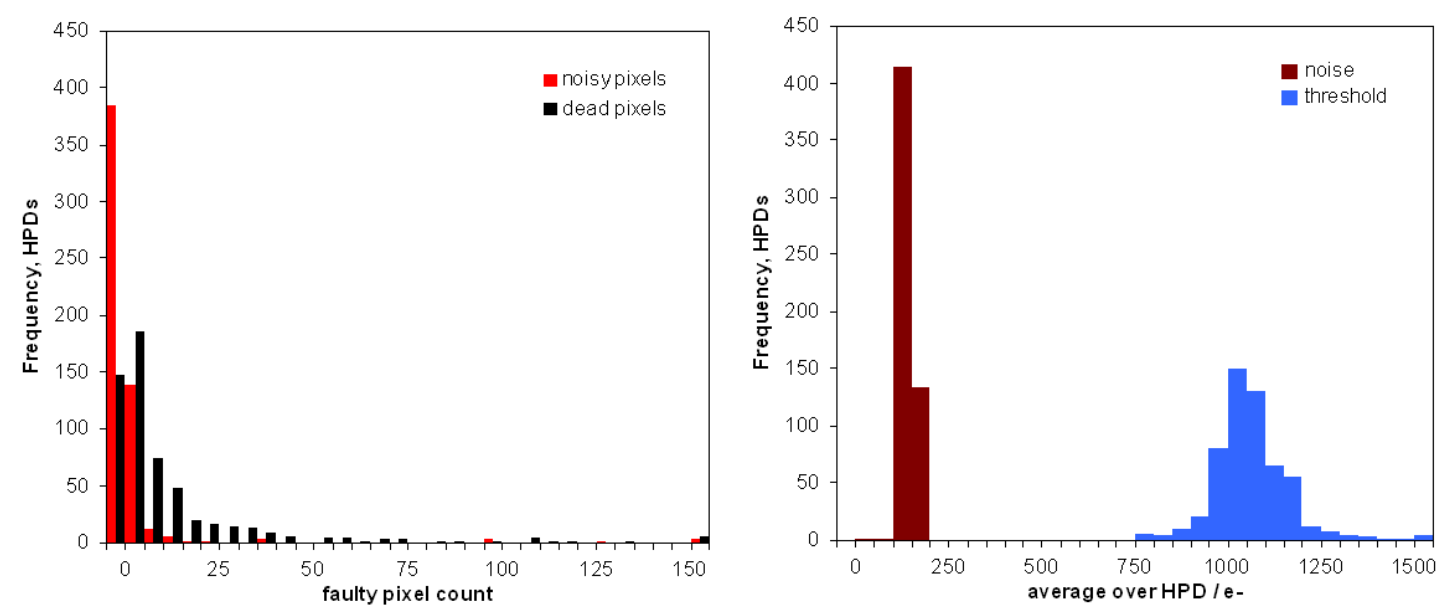

Figure 3: Pixel operation results from production HPDs. Left, the number of dead and noisy channels out of 8192. Right, the average pixel thresholds and noise per HPD, c.f. the typical signal of 5000 e-.

HPDs which fail our selection criteria (Table 2), such that they are unusable in the RICH. This corresponds to a yield of $>97.5 \%$. The summary of results is presented in Table 3, Selected histograms are given in Figs. 2 and 3 . The low level of residual gas in the HPD is primarily helium. We have verified this by examining parasitic gain effects within the HPD as a function of photocathode potential, for example we find a plateau region between $20 \mathrm{~V}$ and $25 \mathrm{~V}$ consistent with the first ionisation energy of Helium, $24.6 \mathrm{eV}$.

We note in particular the ability of Photonis-DEP to consistently improve the QE of the HPDs over the production period, demonstrated in Fig. 4. We have independently confirmed the improvement through the repeated measurements performed at one of the PDTFs. To quantify the improvement we integrate across the energy spectrum. This is demonstrated in Fig. 5, with the summary also in Table 3. We compare this with our expected integrated QE obtained from pre- 

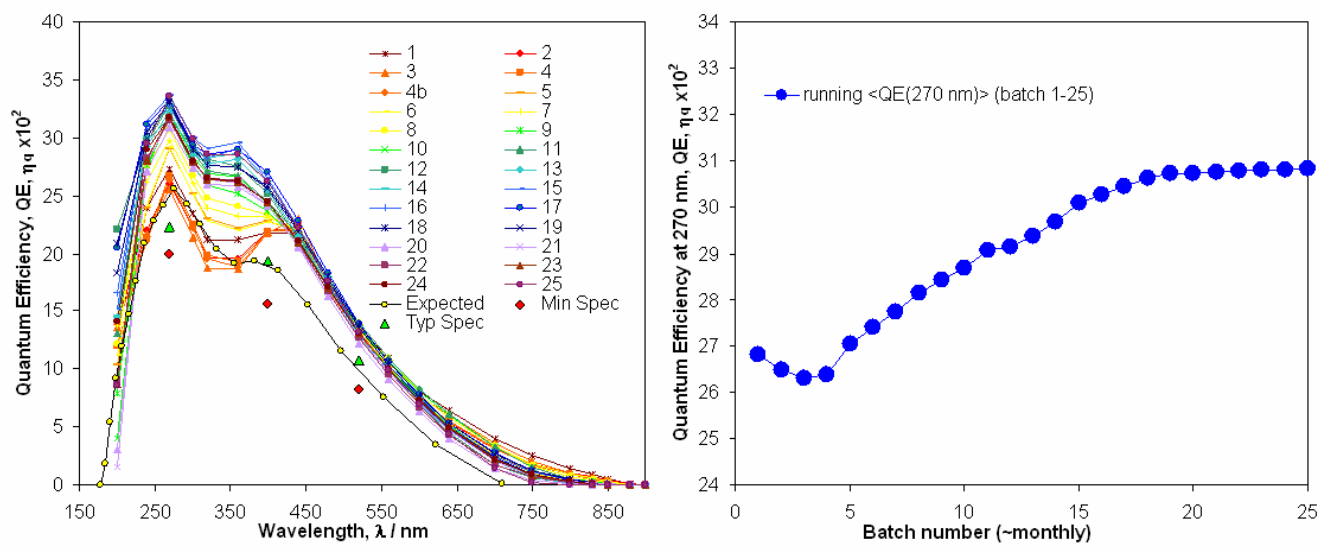

Figure 4: The QE of HPD production batches. Left, average QE per batch, as a function of wavelength. Expectations result from measurements on prototype HPDs. Right, the average peak QE, at $270 \mathrm{~nm}$, as a function of batch.
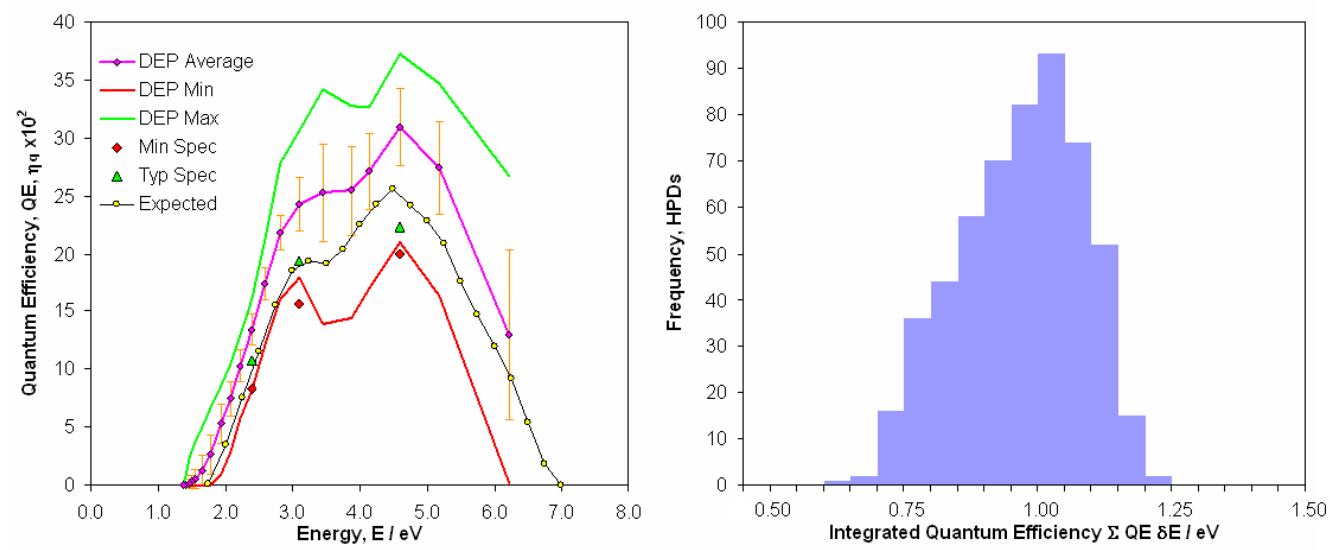

Figure 5: QE as a function of energy. Left, a summary of the entire HPD sample, as a function of photon energy. Vertical bars indicate the standard deviation of the Photonis-DEP QE measurements, no error is included. Red and green solid curves are the extrema of the data at each point. Right, a summary of the entire sample, the integrated $\mathrm{QE}$ in $\mathrm{eV}$ as a function of photon energy.

series and prototype HPDs, of $0.761 \mathrm{eV}$. We see, on average, a $27.1 \%$ improvement.

\section{Discussion}

The structure and implementation of the LHCb RICH detector, including the photo-detectors, have been thoroughly tested in testbeam scenarios. The 288 RICH 2 photo detectors are now installed in the LHCb experiment and have been successfully read out in parallel with the final DAQ system. All of the the HPDs for RICH 1 are now prepared and ready for installation.

We have seen that the HPDs not only meet the specifications of the LHCb RICH, but exceed them in key areas. We have previously demonstrated the radiation tolerance, magnetic field tolerance and lifetime of the HPDs, reported elsewhere [6, 7, 8, 9]. The dark count rate is on average a 
factor of 2000 lower than the specifications and will have a negligible affect on physics in LHCb. The afterpulse rate resulting from ion feedback has been seen to be a factor of thirty times lower than the specified value, indicating high vacuum quality in all production HPDs. The average increase in QE of $27.1 \%$ will significantly increase the number of detected photons within the RICH systems.

\section{Conclusions}

The full sample of pixel hybrid photon detectors required for the LHCb RICHes, including spares and replacements, have been produced. The HPDs have been fully tested by the LHCb collaboration at two custom photo-detector test facilities. We report an accepted yield exceeding $97.5 \%$, with the majority of HPDs exceeding the requirements and/or the specifications in key areas, including the vacuum quality, the inherent dark noise, the pixel chip quality and in particular the quantum efficiency. The LHCb RICH detector will be ready for data taking at the turn-on of the LHC in early 2008.

\section{Acknowledgements}

The LHCb RICH collaboration would like to extend their thanks to their industrial partners, particularly the HPD manufacturer Photonis-DEP, who through continuous work have produced the excellent devices we are pleased to present herein.

\section{References}

[1] S. Amato et al., the LHCb collaboration, LHCb Technical Proposal, CERN-LHCC-98-004 LHCb, 1998.

[2] R. Antunes-Nobrega et al., the LHCb collaboration, LHCb Reoptimised Detector Design and Performance, Technical Design Report 9, CERN LHCC 2003-030 LHCb, 2003.

[3] S. Amato et al., the LHCb collaboration, LHCb RICH, Technical Design Report 3, CERN-LHCC-2000-037 LHCb, 2000.

[4] T. Gys, Nucl. Instr. and Meth. A 567 (2006) 1, pp. 176-179.

[5] M. Campbell et al., IEEE Trans. Nucl. Sc. 53 (2006) 4, pp. 2296-2302.

[6] K. Wyllie et al., Nucl. Instr. and Meth. A 530 (2004) 1-2 pp. 82-86.

[7] N. Kanaya et al., Nucl. Instr. and Meth. A 553 (2005) 1-2, pp. 41-45.

[8] M. Patel, M. Losasso and T. Gys, Nucl. Instr. and Meth. A 553 (2005) 1-2, pp. 114-119.

[9] G. Aglieri Rinella et al., Nucl. Instr. and Meth. A 553 (2005) 1-2, pp. 120-124. 